\title{
Chiropractic Management of a Patient With Neck-Tongue Syndrome: A Case Report
}

\author{
Craig S. Roberts, DC \\ ABSTRACT \\ Objective: The purpose of this case report was to describe the chiropractic management of a patient with neck-tongue \\ syndrome (NTS). \\ Clinical Features: A 34-year-old female patient sought treatment at a chiropractic clinic for symptoms involving neck \\ pain associated with left-sided paresthesia of the tongue that had persisted for $>2$ years. A diagnosis of NTS was made. \\ Intervention and Outcome: The patient was treated with spinal manipulation, myofascial release, and home exercises. \\ After 2 weeks, she was symptom free. At the 2-year follow-up, the patient remained free of symptoms. \\ Conclusion: This patient with NTS responded favorably to a course of chiropractic care. (J Chiropr Med 2016;15:321-324) \\ Key Indexing Terms: Manipulation, Spinal; Paresthesia; Nerve Compression Syndromes; Chiropractic
}

\section{INTRODUCTION}

Neck-tongue syndrome (NTS) presents with unilateral upper neck or occipital pain and altered sensation in the ipsilateral half of the tongue that is aggravated by neck movement. ${ }^{1}$ The prevalence of NTS in adults is estimated to be 2.2 individuals out of $1000 .^{2}$ The paper that reported this figure was based on examination of 1838 individuals aged 18-64 and not on individuals who sought care. The number of patients seeking care for NTS is likely to be lower than 2.2 out of 1000 . The low number of case studies reported in the literature (approximately 50 cases involving individuals aged 6-65 years have been described in the literature ${ }^{3}$ ) and the lack of mention of the syndrome in common orthopedic texts attest to this.

It is unknown whether patients experiencing NTS would report to a health care provider proficient in spinal manipulative therapy (SMT). It also is unknown whether other health care professionals would refer such a case to a provider of SMT. This case report adds to a small body of case reports that suggest that SMT can be effective in the treatment of NTS. Of the peer-reviewed reports available on PubMed, only 3 involved SMT as treatment ${ }^{4-6}$; all reported successful outcomes. Therefore, the purpose of this case report was to describe the management of a patient with NTS.

Private Practice, Nevada City, CA.

Corresponding author: Craig S. Roberts, DC, 582 Searls Ave, Nevada City, CA 95959. Tel.: +1 5304708500.

(e-mail: craig@docroberts.com).

Paper submitted January 16, 2016; in revised form August 5, 2016; accepted August 5, 2016.

1556-3707

(C) 2016 National University of Health Sciences.

http://dx.doi.org/10.1016/j.jcm.2016.08.005

\section{Case Report}

A patient aged 34 years presented to a chiropractic clinic with upper left neck pain that was present $50 \%$ to $75 \%$ of the time. Several times in a week, the neck pain was accompanied by a short burst of symptoms, which she described as "tingling," "shooting," "piercing," and "electric shock-like," on the left side of her tongue. The symptoms were several seconds to 30 seconds in duration. The tongue symptoms typically were elicited by sudden, unguarded rotation of the head to the left side.

The patient worked as a massage therapist and was an avid cyclist. The symptoms were an annoyance because of their unpredictability. The patient initially reported to another chiropractic office, which referred her to the author because of his specialization in neurology.

Initial inspection revealed moderate forward head posture and bilateral internal rotation at the shoulders. Orthopedic tests essentially were normal, with the exception of cervical compression, which caused mild pain in the left suboccipital region and bilateral pain at the cervicothoracic junction. Cervical range of motion in the seated position was within normal limits (WNL) and did not elicit tongue symptoms (instrumentation was not used for range of motion because the ranges were visibly normal and symmetrical). Cervical rotation in the supine position was WNL, but tongue symptoms were elicited and lasted for a few seconds when left rotation was performed rapidly and coupled with 10 to 15 degrees of extension. This test was performed only once because of concerns about injuring the myelin sheath of the portions of the $\mathrm{C} 2$ nerve associated with NTS (Elisevich et $\mathrm{al}^{7}$ previously described a case in which nerve injury to the $\mathrm{C} 2$ nerve root had occurred). Neurologic examination of the upper extremities, including 
deep tendon reflexes and sharp touch, was WNL. A brief cranial nerve examination revealed that visual fields, jaw clenching, sternocleidomastoid strength, tongue strength, facial muscle strength, hearing, facial sharp/dull sensation, and palate elevation were WNL and symmetrical.

Radiographs were obtained to rule out osseous pathology, particularly in the atlantoaxial region. Anteroposterior, anterior-to-posterior open mouth, and lateral views revealed no osseous pathology. The mid-cervical spine had a slight kyphotic curve. A diagnosis of NTS was made on the basis of the classic presentation of the syndrome: unilateral upper neck pain upon rotation of the head to the affected side, with ipsilateral paresthesia in the tongue. ${ }^{1}$

Treatment was initiated on the basis of reports of NTS being successfully treated using SMT. ${ }^{4-6}$ Vectors of force were applied with the same rationale as has been previously reported for cervical radiculopathy, ${ }^{8}$ in which directions of manipulation are chosen on the basis of centralization of symptoms. The $\mathrm{C} 0-2$ segments were not adjusted into left rotation with extension because it had been established that left rotation aggravated tongue symptoms. The $\mathrm{C} 0-2$ segments were placed in a position of slight right lateral flexion. Right lateral flexion was the vector of force, with the objective of decompressing the left $\mathrm{C} 1$ and $\mathrm{C} 2$ nerve roots and providing a fast stretch to the left suboccipital musculature. The segments were challenged with increasing levels of mobilization to ascertain that symptoms were not elicited before a high-velocity, low-amplitude thrust was executed, consistent with chiropractic manipulation (diversified technique). Because symptoms were not precipitated by mobilization, the high-velocity, low-amplitude thrust was performed, and cavitation was achieved without eliciting tongue symptoms.

Ischemic compression was directed bilaterally at trigger points in the suboccipital muscle group to reduce hypertonus. The patient was given 2 home exercises to address the forward head posture. The first exercise targeted the deep cervical flexors and was performed as follows: The patient lay supine on the floor; the chin was tucked and the head was supported by the floor; with the chin tucked, the patient lifted the head approximately $1 \mathrm{~cm}$ off of the floor; and this position was held until the neck and head began to shake, indicating fatigue. The patient performed this exercise twice in the morning and twice in the evening.

In the second exercise, often referred to as the "superman exercise," the patient lay prone, with the arms abducted 270 degrees, and then lifted the arms toward the ceiling to engage the lower trapezius. The patient performed this exercise 4 times in the morning and 4 times at night, with 5 -second holds.

The patient stopped experiencing tongue symptoms after 3 visits, and her neck pain resolved after 5 visits. She was treated 9 times in total, and 2 years later, she has had no recurrence of symptoms. The patient gave consent for publication of this case report.

\section{DISCUSSION}

Although NTS is often described as rare, examination of 1838 Danish parishioners (aged 18-65 years) revealed a prevalence of approximately $0.22 \%$. $^{2}$ Lance and Anthony presented the first 4 case studies in $1980 .{ }^{1}$ They did not discuss treatment in their report, and a conclusive anatomical basis for NTS had yet to be shown. The authors hypothesized that afferent fibers from the lingual nerve traveled via the hypoglossal nerve to the second cervical root. They further proposed that the mechanism of compression of the $\mathrm{C} 2$ root was "some degree of unilateral subluxation of the facets of the atlanto-axial joint."

In 1981, Bogduk reported on the dissection of 5 cadavers that was designed to explore the pathogenesis of NTS. ${ }^{9} \mathrm{He}$ reported that the $\mathrm{C} 2$ roots lie deep within the vertebral canal and are not susceptible to bony compression. He did find that the $\mathrm{C} 2$ dorsal root ganglion is susceptible to compression between the posterior arch of the atlas and the superior articular process of the axis during combined extension and rotation and that the ventral rami of $\mathrm{C} 2$ could be stretched over the atlanteal or axial articular process during rotation. Bogduk argued that the $\mathrm{C} 2$ ventral ramus was the compromised tissue and agreed with Lance and Anthony ${ }^{1}$ that proprioceptive fibers from the lingual nerve return to the $\mathrm{C} 2$ ventral ramus via the cervical plexus and that the $\mathrm{C} 2$ ventral ramus is impacted against the edge of an articular process of the atlantoaxial joint. Bogduk believed that ipsilateral neck pain is caused by abnormal subluxation of that joint rather than by nerve compression because experimental compression of spinal nerves or peripheral nerves has produced numbness but not pain.

Elisevich et al later reported on a surgical case of NTS. ${ }^{7}$ The patient had experienced severe symptoms for a number of years. Radiography demonstrated assimilation of the atlas to the occiput. Flexion or extension films did not show abnormal atlantoaxial motion. The patient did not respond to immobilization with a cervical collar or to pain medication. Surgical exploration revealed that the atlantoaxial articulating process was more protuberant on the symptomatic side than on the asymptomatic side, thereby stretching the $\mathrm{C} 2$ spinal nerve and its rami in a caudal direction. Elisevich et $\mathrm{al}^{7}$ reported that the $\mathrm{C} 2$ spinal rami, especially the ventral rami, showed nerve fiber loss and fibrosis. The $\mathrm{C} 2$ spinal nerves and their dorsal and ventral rami were resected bilaterally. Postoperatively, the patient experienced mild symptoms only when fatigued. Additionally, the patient had permanent hypoesthesia to pinprick in the distribution of both greater occipital nerves.

Cassidy and Diakow ${ }^{4}$ reported 3 cases treated successfully with spinal manipulation. The authors noted that in all previous case reports, only 1 patient had been treated with incomplete success (the surgical patient discussed previously). Cervical radiographs of all 3 of the patients in the study by Cassidy and Diakow revealed normal results (ie, 
no signs of instability or subluxation at the atlantoaxial joints). All patients demonstrated symptoms typical of NTS. The participants were treated daily for 2 weeks with rotary manipulation to the upper cervical spine. All participants were free of symptoms within 2 weeks and at follow-up. The authors further noted that if instability were present, the patients would have been unlikely to be without neck pain after 2 weeks of SMT. All patients presented with hypertonicity of the suboccipital musculature on the affected side. Cassidy and Diakow ${ }^{4}$ suggested that the C2 nerve could be influenced by spasm of the suboccipital muscles, especially the inferior oblique muscle, because the C2 nerve exits beneath that muscle and is attached to it through fascial attachments. Terret described 7 more cases with positive outcomes with the use of SMT as the treatment modality. ${ }^{5}$

Borody ${ }^{6}$ reported significant improvements in NTS symptoms in patients after administration of a course of spinal manipulation. Radiography revealed a narrowed paraodontoid space on the side contralateral to the side with symptoms. The radiology report stated that the asymmetry could have been attributed to the atlas being rotated to the right on the axis. Borody reported that 44 published cases of NTS had been reported and that either the atlantoaxial subluxation model proposed by Bogduk or the inferior oblique spasm hypothesis of Cassidy and Diakow may be accurate, depending on the case presentation and response to treatment. ${ }^{6}$ Borody proposed differentiating NTS into 2 categories: complicated and uncomplicated. In uncomplicated NTS, symptoms appear to be idiopathic or are precipitated by trauma, such as that caused by a motor vehicle collision. Complicated cases are defined as "NTS in association with an underlying disease process, such as severe degenerative joint disease, inflammatory disease (ie, ankylosing spondylitis), and myelopathic conditions." He noted that all of the 13 cases of uncomplicated NTS treated with SMT at that time experienced a significant reduction in symptoms. Others have followed suit in dividing NTS into complicated and uncomplicated subgroups. ${ }^{10}$

Niethamer and Myers reported a case study of a patient with NTS who was treated successfully with manual therapy and home exercises. ${ }^{3}$ Therapy consisted of exercises and stretches, mobilization of joints, and neuromobilization. No SMT was applied. At the 22-month follow-up, the patient reported a reduction in symptoms but continued to have infrequent episodes of tongue dysesthesia and a symptom described as a "momentary tweak" in the neck when turning to the left.

Orrell and Mardsen reported on 2 cases of NTS. ${ }^{11}$ In the first case, computed tomography revealed no abnormalities in the atlantoaxial region, but a radioisotope bone scan did reveal increased uptake in the left atlantoaxial joint. The authors attributed this increase to osteoarthritis. Injection of an anesthetic and a steroid into the area led to brief relief of symptoms. The second case involved a patient with a fracture to the occipital condyle, and treatment and outcome in this case were not discussed.

A series of cases published by Lewis et al suggested an autosomal dominant inheritance pattern in some cases of NTS. ${ }^{12}$ Eight cases, including 5 adolescents and 3 adults (who were parents of the affected adolescents), were presented. Lewis et al noted that patients had normal examination and neuroimaging results.

The present case report adds to the body of literature supporting the use of SMT in Borody's classification of uncomplicated NTS. This case reports complete alleviation of NTS symptoms with treatment consisting of SMT, myofascial release of suboccipital musculature, and postural exercises. Given the 2-year course of the patient's symptoms, the rapidity of apparent success with treatment, and the success of SMT treatment described in previous reports on NTS cases, the author finds it unlikely that the patient's resolution of symptoms was spontaneous, although that is a possibility.

\section{Limitations}

Limitations of this case report include use of a single sample and simultaneous application of 3 treatments (exercise, myofascial release, and SMT). Although large studies may be difficult to orchestrate because of the low number of cases reported since the syndrome was first described in 1980, such a study would shed more light on the efficacy of various treatment modalities in uncomplicated NTS. For this case, the author chose to apply several treatment modalities in an effort to normalize joint function as quickly as possible to relieve the patient's symptoms. If a future study involves a larger sample base, participants could be divided into groups to determine the effect of exercise, myofascial release, and SMT have individually. Last, with a sample of 1 , the natural course of the condition may be responsible for the apparent treatment effects. Although this is possible, the rapid onset of symptomatic relief after initiation of care after a 2-year course of symptoms points to the causative effect of treatment.

\section{CONCLUSIONS}

This patient with NTS responded favorably to chiropractic management. This result suggests that patients with a similar diagnosis may benefit from a course of chiropractic care.

\section{Funding Sources And Conflicts of INTEREST}

No funding sources or conflicts of interest were reported for this study. 


\section{CONTRIBUTORSHIP INFORMATION}

Concept development (provided idea for the research): C.S.R.

Design (planned the methods to generate the results):

\section{C.S.R}

Supervision (provided oversight, responsible for organization and implementation, writing of the manuscript): C.S.R.

Data collection/processing (responsible for experiments, patient management, organization, or reporting data): C.S.R.

Analysis/interpretation (responsible for statistical analysis, evaluation, and presentation of the results): C.S.R.

Literature search (performed the literature search): C.S.R. Writing (responsible for writing a substantive part of the manuscript): C.S.R.

Critical review (revised manuscript for intellectual content, this does not relate to spelling and grammar checking): C.S.R.

\section{Practical Applications}

- Neck-tongue syndrome presents with unilateral neck pain and altered sensation in the ipsilateral tongue that is aggravated by neck movement.

- Neck-tongue syndrome may be considered complicated due to underlying pathology or uncomplicated.

- Uncomplicated NTS may be effectively managed with spinal manipulative therapy.

\section{REFERENCES}

1. Lance JW, Anthony M. Neck-tongue syndrome on sudden turning of the head. J Neurol Neurosurg Psychiatry. 1980;43(2): 97-101.

2. Sjaastad O, Bakketeig LS. Neck-tongue syndrome and related (?) conditions. Cephalalgia. 2006;26(3): 233-240.

3. Niethamer L, Myers R. Manual therapy and exercise for a patient with neck-tongue syndrome: a case report. Orthop Sports Phys Ther. 2016;46(3):217-224.

4. Cassidy D, Diakow P. Treatment of neck-tongue syndrome by spinal manipulation: a report of three cases. Pain Clin. 1986; 1:41-46.

5. Terrett AGJ. Neck-tongue syndrome and spinal manipulative therapy. In: Vernon H ed. Upper Cervical Syndrome: Chiropractic Diagnosis And Treatment. Baltimore, MD: Williams and Wilkins; 1988. p. 223-229.

6. Borody C. Neck-tongue syndrome. J Manip Physiol Ther. 2004;27(5):e8.

7. Elisevich K, Stratford J, Bray G, Finlayson M. Neck tongue syndrome: operative management. J Neurol Neurosurg Psychiatry. 1984;47(4):407-409.

8. Peterson CK, Schmid C, Leemann S, Anklin B, Humphreys BK. Outcomes from magnetic resonance imaging-confirmed symptomatic cervical disk herniation patients treated with high-velocity, low-amplitude spinal manipulative therapy: a prospective cohort study with 3-month follow-up. J Manip Physiol Ther. 2013;36(8):461-467.

9. Bogduk N. An anatomical basis for the neck-tongue syndrome. J Neurol Neurosurg Psychiatry. 1981;44(3): 202-208.

10. $\mathrm{Hu} \mathrm{N}$, Dougherty C. Neck-tongue syndrome. Curr Pain Headache Rep. 2016;20(4).

11. Orrell RW, Mardsen CD. The neck-tongue syndrome. J Neurol Neurosurg Psychiatry. 1994;57(3):348-352.

12. Lewis DW, Frank LM, Toor S. Familial neck-tongue syndrome. Headache. 2003;43(2):132-134. 